\section{Neubildungen an den Stimmlippen interdisziplinär diagnostizieren und therapieren}

S törungen des Stimmklangs wie Heiserkeit oder Resonanzminderung sowie Einschränkungen der stimmlichen Leistungsfähigkeit bzw. Belastbarkeit, aber auch subjektive Missempfindungen können auf gutartige Veränderungen der Stimmlippen hindeuten, erklärte Dr. Susanne Voigt-Zimmermann, Klinische Sprechwissenschaftlerin an der Universitäts-HNO-Klinik Magdeburg.

Dabei kann es sich um angeborene Veränderungen wie kongenitale Zysten handeln. Es kann jedoch auch infolge mechanischer Reizungen zu Entzündungskaskaden kommen, die sich in vaskulären, bindegewebigen und epithelialen Umbauprozessen der Stimmlippen manifestieren. So kann Rauchen oder eine Fehl- bzw. Überlastung zu einem Reinke-Ödem führen. Eine weitere Ursache kann eine Entzündung durch einen laryngopharyngealen
Reflux sein. Virusinfektionen können eine Papillomatose verursachen.

Wegen der hohen Auflösung und guten Erreichbarkeit der zu untersuchenden Regionen stellt die flexible Endoskopie heute das diagnostische Verfahren der Wahl dar, erklärte Prof. Dr. Christoph Arens, Direktor Universitäts-HNO-Klinik Magdeburg. Da gutartige Neubildungen der Stimmlippen, insbesondere sekundär-organische Veränderungen, selten monokausal bedingt sind und mit funktionellen Störungen einhergehen, sollte die Diagnostik interdisziplinär erfolgen und neben der HNO-ärztlichen und phoniatrischen Untersuchung auch eine Stimmfunktionsuntersuchung umfassen [Voigt-Zimmermann S et al.: Laringorhinootologie 2014; 93 (12): 819-30].

Auch die Therapie sollte interdisziplinär erfolgen. Phonochirurgische Eingrif- fe in Form einer Abtragung oder Augmentation bei angeborenen Zysten oder nicht reversiblen sekundär-organischen Veränderungen einerseits und die Stimmfunktionstherapie andererseits sind dabei als komplementäre Verfahren zu betrachten, so Voigt-Zimmermann. Bei Reflux können Protonenpumpeninhibitoren und/oder diätetische Maßnahmen sinnvoll sein. Papillome sind multimodal phonochirurgisch, durch Impfung und diätetische Maßnahmen zu behandeln.

Nicht selten sei bei funktionellen Stimmstörungen die Halswirbelsäule involviert, sagte Arens. Bei zervikogener Dysphonie könne eine manuelle Behandlung hilfreich sein. Auch ein Beckenschiefstand oder Beinlängendifferenzen können eine Rolle spielen. Hier kann gegebenenfalls die Zusammenarbeit mit einem Orthodpäden bzw. Physiotherapeuten sinnvoll sein.

Voigt-Zimmermann S, Arens C: Gutartige Neubildungen der Stimmlippen: Diagnostik und Therapie.

\section{Paradigmenwechsel in der Behandlung bakterieller Atemwegsinfektionen}

D ie Resistenzsituation bei den Antibiotika, die in der HNO-Heilkunde eingesetzt werden, ist nach wie vor günstig. Damit dies so bleibt, ist die Indikation zur systemischen antibiotischen Therapie in der täglichen Praxis streng zu stellen.

Infektionen im Kindes- wie Erwachsenenalter betreffen am häufigsten die Atemwege. In bis zu $90 \%$ der Fälle sind unkomplizierte Atemwegsinfektionen viral bedingt und Antibiotika nicht indiziert. Auch bei manchen bakteriellen Infektionen hat sich die Therapie in den letzten Jahren stark gewandelt und die Indikation zur systemischen antibakteriellen Therapie wird heute sehr streng gestellt, berichtete Dr. Horst Luckhaupt, Leiter der Klinik für Hals-, Nasen- und Ohrenheilkunde am St.-Johannes-Hospital Dortmund.

Die akute Rhinosinusitis wird vorrangig symptomatisch behandelt, beispielsweise mit abschwellenden Nasentropfen. Lediglich bei eitrigen Entzündungen der Nasennebenhöhlen mit starken
Schmerzen, hohem Fieber, beginnenden Anzeichen von Komplikationen sowie bei schweren Grunderkrankungen erfolgt eine Antibiotikatherapie.

Leitkeime der akuten purulenten Sinusitis sind Streptococcus pneumoniae und Haemophilus influenzae, bei akuten Exazerbationen der chronischen Rhinosinusitis Staphylococcus aureus. Behandelt wird beispielsweise mit Amoxicillin, aber auch die Tetrazykline gewinnen bei Infektionen im HNO-Bereich wieder an Bedeutung, so Luckhaupt.

Auch in der Behandlung der akuten Otitis media habe ein Paradigmenwechsel stattgefunden, sagte Luckhaupt. Bei Säuglingen in den ersten sechs Lebensmonaten mit akuter Mittelohrentzündung sollte immer eine antibiotische Therapie erfolgen, bei Kleinkindern bis zum zweiten Lebensjahr lediglich bei ausgeprägten Krankheitszeichen mit starken Schmerzen und hohem Fieber. Kinder ab dem zweiten Lebensjahr sollten bei nicht schwerem Krankheitsbild zunächst sym- ptomatisch mit Analgetika und abschwellenden Nasentropfen behandelt werden, der Verlauf sollte nach spätestens 48 Stunden kontrolliert werden. Kinder mit einem Cochlea-Implantat, die an einer Mittelohrentzündung erkranken, werden stets antibiotisch therapiert, ebenso Kinder mit Risikofaktoren im Rahmen einer schweren Grunderkrankung. Mittel der Wahl ist Amoxicillin, bei Penicillin-Allergie alternativ zum Beispiel ein Makrolid-Antibiotikum.

Bei chronischer Otitis media kann es auch zu einer Superinfektion in Form einer Mykose kommen. Pilzinfektionen des Ohres treten vorwiegend im äußeren Gehörgang auf. Leitkeime sind Aspergillen und Candida albicans. Eine subtile ohrmikroskopische Reinigung und gegebenenfalls Spülung seien hier besonders wichtig, so Luckhaupt. Neben einer gezielten antimykotischen Therapie kann auch mit Farbstoffen behandelt werden, die eine gute antiseptische, antibakterielle und antimykotische Wirkung haben.

Luckhaupt H: Infektionen im HNO-Bereich.

Kongressberichterstattung: Angelika Bauer-Delto 